\title{
The Hall technique is an effective treatment option for carious primary molar teeth
}

\section{Is the Hall technique more effective than conventional methods of caries management for primary molars? Is the Hall technique acceptable to children, their carers and dentists?}

\author{
Innes NP, Evans DJ, Stirrups DR. \\ The Hall technique: a randomized controlled clinical trial of a \\ novel method of managing carious primary molars in general \\ dental practice: acceptability of the technique and outcomes at \\ 23 months. BMC Oral Health 2007, 7:18
}

Design This was a general dental practice (GDP)-based, split-mouth, randomised controlled trial (RCT).

Intervention The Hall technique, where a preformed metal crown (PMC) is cemented with no local anaesthesia, caries removal or tooth preparation, was compared with restoration with the material the GDP would normally choose.

Outcome measure The outcomes were recorded as success; minor failure (restoration failure or reversible pulpitis, which could be managed by repair or replacement); or major failure (signs or symptoms of irreversible pulpal damage, such as dental abscess, or tooth broken down and unfeasible to repair or replace the restoration).

Results A total of 128 conventional restorations were placed on 132 control teeth, and 128 PMC on 132 intervention teeth. Eighty-nine per cent of Hall PMC were rated by dentists as causing no apparent discomfort through to merely mild discomfort: for the control restorations this was $78 \%$ (not a significant difference). The Hall PMC outperformed the control restorations in terms of the number of major and minor failures (see Table 1).

Preference for the Hall technique was recorded at $77 \%$ for the children, $83 \%$ for carers and $81 \%$ for dentists who expressed a preference, which this was significant (Chi square, $P<0.0001$ ).

Conclusions The Hall technique was preferred to conventional restorations by the majority of children, carers and dental practitioners. After 2 years, Hall PMC showed more favourable outcomes for pulpal health and restoration longevity than conventional restorations. The Hall technique appears to offer an effective treatment option for carious primary molar teeth.
Address for correspondence: Nicola P Innes, Dundee Dental Hospital and School, Park Place, Dundee DD1 4HR, Scotland, UK. E-mail: n.p.innes@dundee.ac.uk

\section{Commentary}

The results of this study suggest that general dental practitioners should reconsider their use of traditional or "classical" restorative procedures and materials, in favour of the use of PMC without caries removal. To arrive at this recommendation, the authors conducted a GDP-based, split-mouth RCT to determine if the Hall technique is more effective than conventional methods of caries management for primary molars, and whether it is acceptable to children, their guardians and their dentists.

The Hall technique is a minimally invasive restorative procedure using PMC cemented with no local anaesthesia, no caries removal and no tooth preparation (www.scottishdental.org/pbrn/research/ papers/rt03.htm). The Hall technique was compared with use of the restoration material normally chosen by the general dentist rather than comparison with a single, controlled method, and 17 general dental practices participated in the study. This approach provided comparison of a variety of practice approaches.

The recorded outcomes were success, minor failures (restoration failure or reversible pulpitis which could be managed by repair or replacement) or major failures (signs or symptoms of irreversible pulpal damage, dental abscess or unrestorable broken teeth). The Hall PMC produced significantly fewer major and minor failures versus the control restorations, and also resulted in one-sixth the number of restorations causing discomfort). Thus, the results of the present trial show that sealing in caries in deciduous molars improves pulpal health, and patients benefit from the smaller cavity size, that there no need for local anaesthesia, and it is a less traumatic procedure for the child.

There are some concerns abut this technique. The first is the time taken for each restoration. In 13\% of the Hall PMC, orthodontic separators were used, which necessitates an additional visit to the GDP. A second concern relates to how the Hall technique involves no occlusal reduction before fitting the PMC. This may be associated with a premature contact following cementation and an increase in occlusal vertical dimensions. This may only be a theoretical concern because for all the 129 cases where data were available, the dentists found that an even occlusal contact had re-established at the 1 year recall. Further, in the authors' experience, it equilibrates in a matter of weeks. The ideal is to have the dentists seeing the children 2 weeks after the crowns are fitted to assess the occlusion so that any problems can be assessed. The study reports that no child re-attended their dentist following placement of a Hall PMC with signs or symptoms of occlusal dysfunction, and no child or parent reported difficulty with eating or symptoms of temporomandibular joint dysfunction syndrome when they were directly questioned by their dentist at the 1 year or at 2 year recall. On the other hand, small children are unlikely to report this kind of discomfort, and parents may not take note of these complaints. 
Table 1. Performance of preformed metal crowns versus standard restorations

\begin{tabular}{l|l|l|l|l|l}
\hline & \multicolumn{2}{l|}{ Control restorations } & Hall technique & \\
\cline { 2 - 7 } & Number & Proportion & Number & Proportion & Significance \\
\hline Major failures & 19 & $15 \%$ & 3 & $2 \%$ & $\mathrm{P}<0.001$ \\
\hline Minor failures & 57 & $46 \%$ & 6 & $5 \%$ & $\mathrm{P}<0.001$ \\
\hline Pain & 13 & $11 \%$ & 2 & $2 \%$ & $\mathrm{P} 0.003$ \\
\hline
\end{tabular}

From a wider perspective, PMC now are being recommended as the optimum restoration by the British Society of Paediatric Dentistry for managing primary molar teeth where caries involves two or more surfaces, or where teeth are badly broken down. ${ }^{1}$ Prior to the current trial, other trials demonstrated their effectiveness ${ }^{2,3}$ but a Cochrane review ${ }^{4}$ did not find strong evidence suggesting that PMC are more successful than filling materials for restoring primary molar teeth.

\section{Practice points}

The Hall technique seems to offer an effective, noninvasive treatment option for carious primary molar teeth involving two or more surfaces.

Sealing in caries in deciduous molars using Hall PMC seems to improve pulpal health and patients benefit from the smaller cavity size, no need for local anaesthesia and a less traumatic procedure from the point of view of child behaviour management.

\section{Editor's note}

A clinical manual detailing this technique is available for download at www.scottishdental.org/resources/HallTechnique.htm

\section{Aronita Rosenblatt}

University of Pernambuco, Brazil and The Forsyth Institute Boston, USA.

1. Fayle SA. UK National Clinical Guidelines in Paediatric Dentistry. Stainless steel preformed crowns for primary molars. Int J Paediatr Dent 1999; 9:311-314.

3. Randall RC, Vrijhoef MM, Wilson NH. Efficacy of preformed metal crowns vs. amalgam restorations in primary molars: a systematic review. J Am Dent Assoc 2000; 131:337-343.

4. Roberts JF, Attari N, Sherriff M. The survival of resin modified glass ionomer and stainless steel crown restorations in primary molars, placed in a specialist paediatric dental practice. Br Dent J 2005; 198:427-431.

5. Innes NPT, Ricketts DNJ, Evans DJP. Preformed metal crowns for decayed primary molar teeth. Cochrane Database Syst Rev 2007; issue 1.

Evidence-Based Dentistry (2008) 9, 44-45. doi:10.1038/sj.ebd.6400579 